\title{
A SURVEY ON AGENT-BASED ONTOLOGY ALIGNMENT
}

\author{
Maxim Davidovsky ${ }^{1}$, Vadim Ermolayev ${ }^{2}$ and Vyacheslav Tolok ${ }^{1}$ \\ ${ }^{1}$ Department of Mathematical Modeling, Zaporozhye National University, Zhukovskogo 66, Zaporozhye, Ukraine \\ ${ }^{2}$ Department of Information Technologies, Zaporozhye National University, Zhukovskogo 66, Zaporozhye, Ukraine \\ m.davidovsky@gmail.com,vadim@ermolayev.com,vyacheslav-tolok@yandex.ru
}

\begin{abstract}
Keywords: ONTOLOGY: ONTOLOGY ALIGNMENT: ONTOLOGY MATCHING: ONTOLOGY MAPPING: INTELLIGENT AGENT: MEANING NEGOTIATION
\end{abstract}

\begin{abstract}
Ontologies today are increasingly used as consensual knowledge representations in many distributed applications. However, if a system of knowledge based nodes is decentralized, the ontologies at those nodes differ. Therefore the alignment of knowledge representations is required. One of the promising approaches to solve this heterogeneity is the use of agents for aligning knowledge representations. The paper presents a brief survey of the approaches to agent-based ontology alignment. The analysis of these approaches is grounded on the analysis of the requirements to ontology alignments by typical applications that address semantic heterogeneity in open and decentralized settings.
\end{abstract}

\section{INTRODUCTION}

Nowadays ontologies are used in many applications for knowledge management, e-commerce, information retrieval and sharing, etc. One of the promising approaches in application scenarios that require operating knowledge representations is the use of intelligent software agents capable of processing ontologies in order to achieve specific goals. Typically in open and decentralized systems, such as the Semantic Web (Berners Lee et al., 2001) different agents possess varying ontologies. These semantic differences entail wrong processing or possible misunderstanding between agents. Ontologies may evolve in time - differently at different processing nodes. Moreover, different parties in an application encounter are not aware of the changes occurred in ontology evolution. The heterogeneity of knowledge representations amplified by the distortion caused by uncoordinated changes makes the use of knowledge in distributed intelligent applications a challenging problem. A possible solution is the use of ontology matching that discovers possible mappings between concepts forming respective ontologies (Euzenat and Shvaiko, 2007). The result of matching is ontology alignment.

It may be argued that the challenge mentioned above is artificial and arises only because the scenarios are over-complicated by the use of distributed ontologies. Unfortunately this is not true and ontologies are a means to solve a number of problems such as domain analysis, knowledge sharing and reuse, etc (Bermejo-Alonso et al., 2006), (Huhns and Singh, 1997). Consequently, ontologies are a means to provide a semantic foundation for solving interoperability challenge in open distributed settings, using agent paradigm in particular. A number of proposed solutions is based on the use of a common shared ontology(-ies) that can be instantiated by agents for a particular encounter (Van Aart et al., 2002), (Tamma, Wooldridge and Dickinson, 2002), (Mascardi et al., 2007). The two major uses of ontologies are outlined by Dong, Hussain and Chang (2008).

The first one assumes that ontologies conceptualize the protocols for guiding agents' behaviour in interactions. The problem in this case is the change of interaction protocols to apply new negotiation goals and strategies reflecting the varying nature of environment(s). Different agents can also use different negotiation terms causing ambiguity or misinterpretation of communicated content in interactions.

Consequently the second case is the use of ontologies as shared vocabularies for resolving content heterogeneity problems in agents communications, for example to carry out translations between ontologies (Obitko, 2007).

A typical difficulty however is that a central or shared ontology or vocabulary is rarely available. 
So, the communicating nodes in a decentralized system have to align their knowledge representations and respective interpretations. Many of the published solutions rely on the existence of a centralized authority that provides such aligned interpretations. In agent-based approaches a specialized agent offering an ontology alignment service is typically involved (e.g. (Cardoso, Teixeira and Oliveira, 2008)). However, it is typically considered that the ontology mappings leading the required alignment are somehow available off the shelf.

Interestingly, ontology alignment in its essence is the problem that has the same nature as the interoperability challenge in open and decentralized systems. So, it may be expected that it may be solved using a similar approach - i.e. having a good solution technique for ontology alignment will facilitate solving the mentioned interoperability challenge. In this paper the frameworks and existing agent-based solutions for ontology alignment are surveyed. The paper starts with the explicit formal definition of ontology alignment. It continues with the analysis of the known challenging applications that involve semantic heterogeneity and require interoperable solutions. Our focus in examining those cases is the severity of the requirement of using the alignments of knowledge representations. Finally, the paper overviews and analyses the agentbased frameworks that aim to solve ontology alignment. Some conclusions on the state of the art in the field are drawn out of those analyses.

\section{ONTOLOGY ALIGNMENT AND APPLICATIONS}

The analysis of the literature on ontology alignment reveals some terminological ambiguity. Some authors e.g. Abolhassani, Hariri and Haeri (2006), Euzenat (2004a) denote ontology alignment as a process of finding correspondences between ontology entities. Other publications e.g. Euzenat and Shvaiko (2007), Gargantilla and Gomez-Perez (2004) define this process as ontology matching and regard alignment as a result of matching. The key concept of "ontology" is also denoted in different ways depending on the viewpoint. In our research the following notions are used.

Following Euzenat and Shvaiko (2007), an ontology is formally denoted as a tuple $o=\langle C, R, I, T, V, \leq, \perp, \in,=\rangle$ where $C$ is the set of concepts (or classes); $R$ is the set of relations (object and datatype properties); $I$ is the set of individuals; $T$ is the set of datatypes; $V$ is the set of values; $\leq$ is a reflexive, anti-symmetric and transitive relation on $(C \times C) \cup(R \times R) \cup(T \times T)$ called specialization, that form partial orders on $C$ and $R$ called concept hierarchy and relation hierarchy respectively; $\perp$ is an irreflexive and symmetric relation on $(C \times C) \cup(R \times R) \cup(T \times T)$ called exclusion; $\in$ is a relation over $(I \times C) \cup(V \times R)$ called instantiation; = is a relation over $I \times P \times(I \cup V)$ called assignment; (the sets $C, R, I, T, V$ are pairwise disjoint).

Hereby, ontology matching is denoted as a process of finding correspondences (or mappings) between the elements of $C, R, I, T, V$. Mapping (or mapping rule (Euzenat and Shvaiko, 2007)) is a tuple $m=\left\langle e, e^{\prime}, \Re, n\right\rangle$, where: $e, e^{\prime}$ are the elements of $C, R, I, T$ or $V$ of respective ontologies $o$ and $o^{\prime} ; \mathfrak{R}=\{\subset, \subseteq, \equiv, \supset, \supseteq\}$ is a set of relations; and $n$ is a confidence value (typically in the range of $[0,1]$ ).

According to the specification of OWL (www.w3.org/standards/techs/owl\#w3c_all), that is a de facto standard ontology representation language $e$ and $e^{\prime}$ are represented in OWL as classes, datatypes, object properties, data properties, annotation properties or named individuals.

A good survey of ontology-based applications is (Gargantilla and Gomez-Perez, 2004). The applications of agent-based ontology alignment are surveyed by Euzenat and Shvaiko (2007), ontology matching applications in particular. A comprehensive summary of ontology matching techniques and applications is (Scharffe et al., 2007). Based on these we figure out the following several typical applications, specifically those using agent orientation, and analyse to which extent they require ontology alignment.

Information retrieval and knowledge sharing: A number of proposals address Information retrieval (IR) supported by intelligent software agents - for example (Zuo, 2006), (Mohammadian and Jentzsch, 2004), (Finin et al., 2005).

A well founded framework based on a multiagent system for querying heterogeneous data sources integrated using ontologies was developed in the SEWASIE project (Dongilli, Fillottrani, Franconi and Tessaris, 2005). In IR intelligent software agents are used for extracting information or knowledge satisfying the semantics and the context of a user query. Alignments are needed for correlating query structure and semantics with information resource schemas and metadata. The critical characteristic here is high recall as it is important not to miss any potentially relevant 
information while non-relevant can be sifted out in subsequent steps.

Data/ information integration: Ontology alignments are used in this application for integrating data stored in separate, partial and heterogeneous sources into a single asset. Integration of the schemas of different databases can also be regarded in this category. A multi-agent architecture for data integration based on ontologies is presented in (Medcraft, Schiel and Baptista, 2003). A method for specifying schema mappings and agents actions in XML data integration task is described in (Brzykcy et al., 2008). A detailed problem statement and solution is elaborated in (Nagy and VargasVera, 2010). For this kind of applications the degree of automation is very important as data/information integration is a laborious task. Besides the effort to be spent, the involvement of a human in the loop may be the cause of errors.

Dynamic data/information fusion: Agentbased solutions are used in fusion scenarios for dynamic creation of semantically adequate samples from heterogeneous sources. (Sobh, 2009) proposes a multi-agent model for carrying out information fusion from multiple sensors in dynamic environment. An in-depth analysis of the information fusion problem together with respective solutions and implementations in the field of geographical information processing are presented in (Duckham and Worboys, 2007). As argued by these authors, the critical parameters for those solutions are speed and level of automation as information fusion is usually carried out in real-time (e.g. signal processing from multiple sensors).

Human-machine dialogues: Alignments are used in human-agent interaction to provide mutual understanding between a user and an agent. They can be used for intelligent human-machine dialoguing in order to obtain a formalizable set of requirements, structures, queries, etc. from informal or poorly structured user descriptions. As a rule such dialogs are run in iterative way. (Brasoveanu et al., 2010) argue the importance of using generic multimodal ontologies on the Semantic Web and propose an approach to enhance human-agent interaction based on multimodal ontologies. (Guzzoni et al., 2007) propose a toolkit-based approach for modeling human-agent interaction. Their toolset provides a means to model different aspects of an intelligent assistant such as: ontologybased knowledge structures; service-based primitive actions; composite processes and procedures; natural language and dialog structures. (Tijerino et al., 2004) report a framework for human-agent collaboration for the purpose of problem solving on the Semantic Web. In human-machine dialogue scenarios the most critical features are adaptability, integrativity, and scalability that allow enhancing human-machine mutual understanding.

Ontology evolution, versioning, refinement, instance migration: Ontology evolution and refinement are the necessary conditions for adequately representing knowledge for dynamically changing domains. Agents use ontology matching to determine the knowledge that remains adequate to the changes for reuse in a new ontology version or in a new ontology. A typical subtask is instance migration in order to populate the obtained target ontology ABox based on the analysis of the structural differences between the sorce and the target TBoxes (Davidovsky, Ermolayev and Tolok, 2010). Packer, Gibbins and Jennings (2009) present an approach for ontology evolution and knowledge acquisition based on agent collaboration. The approach enables agents augmenting their ontologies by selecting and sharing the fragments of ontologies that correspond to a particular domain of interest, or even narrower - to a particular concept. The highlights of the approach are complexity and concept acquisition cost reduction by sharing only those concepts and relationships that relate to a particular case. The agent-based approach for ontology evolution is presented in ( $\mathrm{Li}$ and Yang, 2008) where the process of ontology refinement is driven by negotiation rounds among agents. The approach is applied to supply chain case study. Similarly to data/information integration task, the importance of a high level of automation and decreasing human effort is emphasised for this kind of applications.

Web service composition: Agents draw up compositions of services conforming the requirements and privileges of a user or an agent. Agents use alignments between ontologies describing service interfaces (or profiles) in order to compose web services by connecting their interfaces. The aspects of ontology reconciliation with respect to Web services and their composition are elaborated in (Li and Yang, 2008), (Paurobally, Tamma and Wooldridge, 2007), (Huang, Zavala, Mendoza and Huhns, 2005). An important requirement for such systems is the capability of adaptation and integration for providing compliant access and making the use of aggregate and atomic services more convenient.

The requirements for the described typical software applications that require ontology alignment are summarized in table 1. The characteristics along which the requirements are assessed are implementation independent. In the next section we focus on the solutions of the ontology alignment problem that use agent orientation. The plethora of non-agent oriented approaches have been surveyed by other authors - 


\begin{tabular}{|c|c|c|c|c|c|c|c|c|}
\hline Characteristics & $\begin{array}{l}\text { Dू. } \\
\text { कू }\end{array}$ & 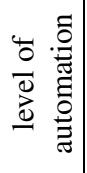 & 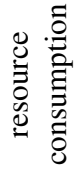 & 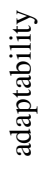 & 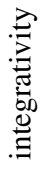 & 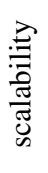 & 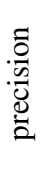 & $\begin{array}{l}\bar{\Xi} \\
\stackrel{\circlearrowright}{0}\end{array}$ \\
\hline Information retrieval and knowledge sharing & $\mathrm{n}$ & $\mathrm{c}$ & $\mathrm{a}$ & $\mathrm{u}$ & $\mathrm{n}$ & $\mathrm{a}$ & $\mathrm{a}$ & $\mathrm{c}$ \\
\hline Data/information integration & $\mathrm{u}$ & $\mathrm{c}$ & $\mathrm{u}$ & $\mathrm{n}$ & $\mathrm{n}$ & $\mathrm{a}$ & $\mathrm{c}$ & $\mathrm{c}$ \\
\hline Dynamic data/information fusion & $\mathrm{n}$ & $\mathrm{c}$ & $\mathrm{a}$ & $\mathrm{n}$ & $\mathrm{n}$ & $\mathrm{n}$ & $\mathrm{a}$ & $\mathrm{n}$ \\
\hline Ontology evolution, refinement, instance migration & $\mathrm{u}$ & $\mathrm{c}$ & $\mathrm{u}$ & $\mathrm{a}$ & $\mathrm{a}$ & $\mathrm{a}$ & $\mathrm{c}$ & $\mathrm{c}$ \\
\hline Human-machine dialogues & $\mathrm{n}$ & $\mathrm{a}$ & $\mathrm{n}$ & $\mathrm{c}$ & $\mathrm{c}$ & $\mathrm{c}$ & $\mathrm{a}$ & $\mathrm{a}$ \\
\hline Web service composition & $\mathrm{n}$ & $\mathrm{c}$ & $\mathrm{u}$ & $\mathrm{c}$ & $\mathrm{c}$ & $\mathrm{a}$ & $\mathrm{c}$ & $\mathrm{c}$ \\
\hline
\end{tabular}

Table 1: Requirements for ontology alignment in typical applications in open and distributed settings. (Legend: $\mathrm{c}-\mathrm{crucial}$, $\mathrm{n}$ - necessary, $\mathrm{a}$ - advisable, $\mathrm{u}$ - uncritical)

for example (Chuttur, 2011), (Vázquez-Naya et al., 2009), (Zhdanova et al., 2004), (Euzenat and Shvaiko, 2007), (Euzenat et al., 2004b).

\section{AGENT-BASED SOLUTIONS FOR ONTOLOGY ALIGNMENT}

Many influential publications, for example (Berners Lee et al., 2001), envision that intelligent software components, like agents, need to be used together with ontologies for making semantic technologies accepted and effective in open and decentralized scenarios. For such agent based solutions, comprising industrial applications, the heterogeneity problem is the challenge that has to be faced. Ontology alignments are a means to solve the challenge. The problem has received substantial attention in the literature. In this section the frameworks aiming at solving ontology alignment problem are surveyed. Attention is paid to the basic theoretical formalism and the fitness of the solution for the applications summarized in table 1 .

(Schorlemmer et al., 2007) presents a formal foundation for ontology alignment regarding it as a product of meaning negotiation between intelligent software agents. The focus is the introduction of general alignment interaction models. The approach is grounded on Barwise and Seligman's theory of information (Barwise and Seligman, 1997) and uses their notion of information flow as a basic formalism. Alignment is defined as a system of classifications and infomorphisms and obtained by meaning coordination between agents $A g_{1}$ and $\mathrm{Ag}_{2}$ through the information channel $A_{1} \stackrel{f_{1}}{\longrightarrow} C \stackrel{f_{2}}{\longleftarrow} A_{2}$, where $C$ is the classification determined by the meaning coordination done before; $A_{1}, A_{2}$ - respective classifications; $f_{1}, f_{2}$ - respective infomorphisms. The IF-based approach has been implemented as the IF-Map method for automated ontology mapping (Kalfoglou and Schorlemmer, 2002).

Atencia and Schorlemmer (2008) argue that semantic alignment in most cases is strongly relative to a particular interaction between agents and even more strongly depends on a particular state of this interaction. Hence, the context of an interaction should be taken into account. This observation is also concurred by a number of approaches addressing the problem (e.g. (Besana and Robertson, 2006)). Information flow theory is sufficiently general and abstract to be applied in almost all application scenarios.

A variety of alternative approaches are grounded on Argumentation Frameworks (AF) introduced in (Dung, 1995) and widely adopted in Artificial Intelligence in broad (Rahwan and Simari, 2009) and in Multi-Agent Systems (MAS) applications (Maudet, Parsons, and Rahwan, 2007). Argumentation is used for ontology alignment by agents to determine acceptable mappings in negotiations. Argumentation-based solutions for negotiations between agents using different ontologies are considered in (Euzenat et al., 2006) with a focus on presenting an argumentation framework for arguing about ontology alignments.

Dung defined argumentation framework as a pair $A F=\langle A R$,attacks $\rangle$ where $A R$ is a set of arguments, attacks is a binary relation on $A R$ and $\operatorname{attacks}(A, B)$ signifies that argument $A$ attacks argument $B$. (Bench-Capon, 2003) extends it to the Value-based AF (VAF) as a 5-tuple $V A F=\langle A R$, attacks,$V$, val,$P\rangle$ where $V$ is a nonempty set of values, val is a function which maps the elements of $A R$ to the elements of $V$ and $P$ is the set of possible audiences. An audience represents one ordered set of values that states if an attack succeeds or fails according to the values the arguments promote. The use of VAF allows prescribing assigning different strengths to arguments depending on the values and accounting for different interests and preferences over 
arguments with respect to a particular agents audience. VAF is used as a common ground for the approaches described in (Laera, Tamma, Euzenat, Bench-Capon and Payne, 2006), (Trojahn, Moraes, Quaresma and Vieira, 2008), and (Isaac et al., 2008). In (Trojahn et al., 2008) VAF is used for alignment compositions and is complemented with a set of confidence degrees and a mapping of those degrees to arguments representing the confidence of an agent in some argument. (Isaac et al., 2008) present a Voting-based VAF (V-VAF) and a Strength VAF (S-VAF). S-VAF extends the VAF with a function that maps elements of $A R$ to real values within $[0,1]$ representing the strength of the argument. V-VAF is defined by adding a notion of support (a reflexive binary relation over $A R$ disjoint to attacks) which allows counting arguments as defenders (or coattackers) within a particular attack. Taking into consideration the obtained counts, voting allows determining whether an attack is successful or not. An interesting comparison of AFs is presented in (Trojahn et al., 2009) which analyzes VAF, S-VAF and V-VAF and evaluates them using ontologies from the Ontology Alignment Evaluation Initiative (OAEI, oaei.ontologymatching.org) evaluation data set. (Maio and Silva, 2010) propose an approach based on the Bipolar AF (BAF) by (Cayrol and Lagasquie-Schiex, 2005). Similarly to V-VAF, BAF extends the AF with the capability of representing the support relation between arguments. In BAF the support relation allows indicating the arguments which are assumed to be independent of the attack relation. (Trojahn and Euzenat, 2010) presents both theoretical and empirical study of ontology consistency state depending on argumentationgrounded alignments. Comparing to approaches based on IF-theory, AF-based ones better fit to those application scenarios where the agent paradigm is commonly used - in information retrieval, humanmachine dialogues and web-service composition. However, it is a promising approach also for such tasks as ontology evolution, refinement and instance migration especially in decentralised settings where manipulating of distributed heterogeneous ontologies is a necessary subtask.

(Ermolayev et al., 2005) elaborate a strategy for automated meaning negotiation. Similarly to (Atencia and Schorlemmer, 2008) their approach aims at aligning ontologies by parts (contexts) that are relevant to a particular negotiation encounter. Negotiations imply iterative reduction of semantic distance between the contexts. An agent uses propositional substitutions which may reduce the distance and support them with argumentation. The process is stopped when the distance reaches a commonly accepted threshold or the involved parties exhaust their propositions and arguments. As opposed to the above-mentioned AF-based approaches this framework addresses the entire process of semantic reconciliation between ontologies and does not require off-the-shelf mappings. The approach is oriented to a specific task of meaning coordination between a query submitter and a mediator agent in distributed information retrieval. However, essentially it does not use any application dependent features and could therefore be used in other applications of ontology alignment.

In a summary it has to be noted that the majority of agent-based solutions use negotiation techniques as the most natural and well-proven mechanism for agent interaction. Several basic theoretical approaches with different expressive power are exploited. However the most widely used formalism is the Dung's Argumentation Framework (or its derivatives).

\section{CONCLUSION}

The paper presented a brief survey of the approaches to agent-based ontology alignment. The analysis of these approaches is grounded on the analysis of the requirements to ontology alignments by typical applications that address semantic heterogeneity in open and decentralized settings.

All the solutions that have been surveyed are rather abstract ones that can be used for different applications than they are application dependent.

The majority of the reviewed frameworks still wait for their implementation and experimental validation. Trojahn (2009) and Isaac (2008) report their experimental setups and evaluation results. Kalfoglou (2002) also reports implementation and application case study. With respect to the rest of the frameworks it is hard to assess and compare the fitness of their approaches to the range of typical applications of ontology alignment.

\section{ACKNOWLEDGEMENT}

The research presented in this paper has been supported in part by DataArt. The company has provided the travel grant for attending and presenting at ICAART 2012.

\section{REFERENCES}

Abolhassani, H., Hariri, B. B., Haeri, S.H. (2006). On Ontology Alignment Experiments. Webology, 3(3). 
Atencia, M., Schorlemmer, M. (2008). Formalising interaction-situated semantic alignment: The communication product. In Tenth International Symposium on Artificial Intelligence and Mathematics (ISAIM'08), Fort Lauderdale, Florida, USA.

Barwise, J., Seligman, J. (1997). Information Flow: The Logic of Distributed Systems. Cambridge University Press.

Bench-Capon, T.J.M. (2003). Persuasion in Practical Argument Using Value-based Argumentation Frameworks. J Logic Computation, (Vol. 13(2003), pp. 429-448)

Bermejo-Alonso, J. et al. (2006). A Survey on Ontologies for Agents. From Theory to Practice. ASLab-ICEA-R2006-002.

Berners-Lee, T. et al. (2001). The Semantic Web. Scientific American, May 2001, p. 29-37.

Besana, P., Robertson, D. (2006). Probabilistic dialogue models for dynamic ontology mapping. In da Costa, P. et al. (Eds.), URSW '06 Uncertainty Reasoning for the Semantic Web (Vol. 2). Proc. $2^{\text {nd }}$ ISWC Workshop on Uncertainty Reasoning for the Semantic Web, CEURWS (Vol. 218).

Brasoveanu, A. et al. (2010). Generic Multimodal Ontologies for Human-Agent Interaction. Int. J. of Computers, Communications \& Control, (Vol. V (2010), No. 5, pp. 625-633).

Brzykcy, G. et al. (2008). Schema Mappings and Agents' Actions in P2P Data Integration System, J. UCS, (Vol. 14(7), pp. 1048-1060).

Cardoso, H. L., Teixeira, D. D., Oliveira, E. (2008). An Ontology-Mapping Service for Agent-Based Automated Negotiation. Proc. AAMAS'08 Workshop on Agent-based Technologies and Applications for Enterprise Interoperability (ATOP).

Cayrol, C., Lagasquie-Schiex, M. (2005). On the Acceptability of Arguments in Bipolar Argumentation Frameworks. Symbolic and Quantitative Approaches to Reasoning with Uncertainty, 378-389.

Chuttur, M. Y. (2011). Challenges Faced by Ontology Matching Techniques: Case Study of the OAEI Datasets. Journal of Information Technology, (Vol.3(1), pp. 33-42).

Davidovsky, M., Ermolayev, V., Tolok, V. (2010). Evaluation of Semi-Automated Ontology Instance Migration. In M. Essaaidi et al. (Eds.), Intelligent Distributed Computing IV, SCI 315 (pp. 179-190), Springer-Verlag Berlin Heidelberg.

Dong, H., Hussain, F. K., Chang, E. (2008). State of the Art in Negotiation Ontologies for MAS. International Journal of Web Services Practices (Vol. 3, No.3-4, pp. 157-163).

Dongilli P., Fillottrani P.R., Franconi E., Tessaris S. (2005). A multi-agent system for querying heterogeneous data sources with ontologies. Proc. of Thirteenth Italian Symposium on Advanced Database Systems, SEBD-2005.

Duckham, M., Worboys, M. (2007). Automated geographic information fusion and ontology alignment. In Belussi, A., Catania, B, Clementini, E., and Ferrari,
E. (Eds.), Spatial data on the Web: Modelling and Management, Chapter 6 (pp. 109-132). Springer, Berlin.

Dung, P. (1995). On the acceptability of arguments and its fundamental role in nonmonotonic reasoning, logic programming and n-person games. Artificial Intelligence, (Vol. 77(2), pp. 321-357).

Ermolayev, V. et al. (2005). A strategy for automated meaning negotiation in distributed information retrieval. In Proc. 4th ISWC, Galway (IE), 201-215.

Euzenat, J. (2004a). An API for ontology alignment. The Semantic Web - ISWC 2004, 698-712.

Euzenat, J. et al. (2004b). State of the art on ontology Alignment. Deliverable D2.2.3. KWEB/2004/D2.2.3/v1.2.

Euzenat J. and Shvaiko P. (2007). Ontology Matching, Berlin Heidelberg (DE), Springer-Verlag.

Euzenat, J. et al. (2006). Negotiation and argumentation techniques among agents complying to different ontologies. Deliverable D2.3.7, KWEB, v1.0.

Finin T. et al. (2005). Information Retrieval and the Semantic Web. Proceedings of the 38th Annual Hawaii International Conference on System Sciences, HICSS '05, 113a-113a.

Gargantilla, J., Gomez-Perez, A. (2004). OntoWeb: A Survey on Ontology-Based Applications. Deliverable 1.6. OntoWeb Consortium IST Project IST-2000-29243

Guzzoni, D., Baur, C., Cheyer, A. (2007). Modeling Human-Agent Interaction with Active Ontologies. Artificial Intelligence SS-07-04, 52-59.

Huang, J., Zavala, R., Mendoza, B., Huhns, M.N. (2005). Reconciling Agent Ontologies for Web Service Applications. Multiagent System Technologies: Third German Conference (MATES-05). (Vol. LNAI 3550 pp. 106 - 117), Berlin, Springer Verlag.

Huhns, M. N., Singh, M. P. (1997). Ontologies for Agents. IEEE Internet Computing, (Vol. 1, no. 6, pp. 81-83).

Isaac, A. et al. (2008). Using quantitative aspects of alignment generation for argumentation on mappings. In Proc. ISWC'08 Workshop on Ontology Matching, Karlsruhe, Germany.

Kalfoglou, Y., Schorlemmer M. (2002). IF-Map: An Ontology-Mapping Method based on Information-Flow Theory, Proc. 1st Int. Conf. Ontologies, Databases and Application of Semantics (ODBASE'02), Irvine, CA, USA

Laera, L., Tamma, V., Euzenat, J., Bench-Capon, T., Payne T. (2006). Reaching agreement over ontology alignments. In Proc. The 5th International Semantic Web Conference (ISWC 2006), Athens, GA, USA.

Li, L., Yang, Y. (2008). Agent negotiation based ontology refinement process and mechanisms for service applications. Service Oriented Computing and Applications 2, 15-25.

Maio P., Silva, N. (2010). Ontology Alignment Argumentation with Mutual Dependency Between Arguments and Mappings. International Workshop on Data Engineering meets the Semantic Web (DESWeb), 26th IEEE International Conference on Data Engineering (ICDE); Long Beach, (CA), USA. 
Mascardi, V. et al. (2007). Enhancing Communication inside Multi-Agent Systems - An Approach Based on Alignment via Upper Ontologies. Proc. Int'l Workshop Agents, Web-Services and Ontologies: Integrated Methodologies (MALLOW-AWESOME '07), 92-107.

Maudet, N., Parsons, S., Rahwan, I. (2007). Argumentation in Multi-Agent Systems: Context and Recent Developments. In Proc. Argumentation in MultiAgent Systems (ARGMAS06). Post-Proc. The Third International Workshop, (Revised), LNCS 4766, Springer-Verlag, 1-16.

Medcraft, P. S., Schiel, U., Baptista C.S. (2003). DIA: Data Integration Using Agents. International Conference on Enterprise Information Systems - ICEIS, 79-86.

Mohammadian M., Jentzsch R. (2004). Chapter II: Computational Intelligence Techniques Driven Intelligent Agents for Web Data Mining and Information Retrieval in Masoud Mohammadian (ed.) Intelligent Agents for Data Mining and Information Retrieval, IDEA Group Publishing, Hershey.

Nagy, M., Vargas-Vera, M. (2010). Towards an Automatic Semantic Data Integration: Multi-agent Framework Approach. In Gang Wu (Eds.) Book chapter in Semantic Web (pp. 107-134). In-Tech.

Obitko, M. (2007). Translations between Ontologies in Multi-Agent Systems. PhD thesis, Czech Technical University in Prague, Czech.

Packer, H.S., Gibbins, N., Jennings, N.R. (2009). Ontology evolution through agent collaboration. Brain $1-4$.

Paurobally, S., Tamma, V., Wooldridge, M. (2007). A Framework for Web service negotiation. ACM Transactions on Autonomous and Adaptive Systems (TAAS), (Vol. 2(4)).

Rahwan, I., Simari, G. R. (2009). Argumentation in Artificial Intelligence, (Eds.). Springer.

Scharffe, F. et al. (2007). Analysis of knowledge transformation and merging techniques and implementations. KWEB/2004/D2.2.7/0.8.

Schorlemmer, M. et al, (2007). A formal foundation for ontology-alignment interaction models. International Journal on Semantic Web and Information Systems (Vol. 3(2), pp.50-68).

Sobh T. (2009). Information Fusion Using OntologyBased Communication between Agents. International Arab Journal of e-Technology, (Vol. 1, No. 2).

Tamma, V., Wooldridge, M., Dickinson, I. (2002). An ontology for automated negotiation. In Proc. The international workshop on ontologies in agent systems (OAS 02), AAMAS 02 conference, Bologna, Italy.

Tijerino, Y.A., Al-Muhammed, M., Embley, D.W. (2004). Toward a flexible human-agent collaboration framework with mediating domain ontologies for the semantic web. In Proc. The ISWC 2004 Workshop on Meaning Coordination and Negotiation (pp. 131-142) Hiroshima, Japan.

Trojahn, C. Euzenat, J. (2010). Consistency-driven argumentation for alignment agreement. In Proc. The Fifth International Workshop on Ontology Matching
(OM-2010) collocated with the 9th International Semantic Web Conference (ISWC-2010) Shanghai, China.

Trojahn, C. et al. (2009). Comparing Argumentation Frameworks for Composite Ontology Matching. Journal on Data Semantics - JODS (Vol. 10, pp. 237263). Springer-Verlag Berlin, Heidelberg.

Trojahn, C., Moraes, M., Quaresma, P., Vieira, R. (2008). A cooperative approach for composite ontology mapping. LNCS 4900, 237.Van Aart, C. et al. (2002). Creating and Using Ontologies in Agent Communication. Telecom Italia EXP magazine (Vol 2, No 3).

Vázquez-Naya, J.M. et al. (2009). Ontology Alignment Overview. Encyclopedia of Artificial Intelligence 2009, (pp. 1283-1289).

Zhdanova A.V. et al. (2004). Ontology Alignment Solution. Deliverable D14 v2.0.

Zuo, L. A. (2006). Semantic and Agent-Based Approach to Support Information Retrieval, Interoperability and Multi-Lateral Viepoints for Heterogeneous Environmental Databases. PhD Thesis, The Department of Electronic Engineering, Queen Mary, University of London. 